\title{
Anti-VEGF therapy: higher potency and long-lasting antagonism are not necessarily better
}

\author{
Ayumi Usui-Ouchi and Martin Friedlander \\ Department of Molecular Medicine, The Scripps Research Institute, Division of Ophthalmology, Scripps Clinic, Lowy Medical Research Institute, La Jolla, California, USA.
}

$T_{\text {he }}$ he "golden years" are much less so if you lose your central vision to neovascular age-related macular degeneration (AMD). Approximately 15 out of 1000 individuals over age 75 will have advanced AMD; $66 \%$ will have the neovascular form that is associated with central vision loss. The introduction of antiVEGF treatment for neovascular AMD 15 years ago (1) has revolutionized care for these patients, much as pan-retinal photocoagulation (PRP) did for patients with proliferative diabetic retinopathy (PDR) and ischemic retinal vein occlusions (RVOs) decades earlier. The difference between these two therapeutic advances is that PRP reduces the ischemic drive by destroying portions of the peripheral retina while VEGF antagonists do little to relieve underlying hypoxia. In addition to having potent vasopermeability and some angiogenic activity, VEGF is an important neurovasculotrophic factor that is critical to the survival and function of neurons and endothelial cells. Because neurovascular cell functions are already compromised by underlying disease (e.g., AMD, diabetes, RVOs), potent, long-lasting VEGF antagonism may be detrimental to the health of cells dependent on its trophic activity. Patients with ischemic (e.g., diabetes, ARMD) retinopathies are likely to have ischemia elsewhere (e.g., heart, brain, kidneys), and the sustained presence of potent VEGF antagonists may prevent adequate collateralization and function in these tissues as well. While the less potent and long-lasting VEGF antagonist drugs may be "just bad enough," newer therapies that are far more potent and/or longer lasting may precipitate problems from suppressed VEGF activity. We will discuss issues to consider when treating ocular disease with VEGF antagonists.

\section{Physiological importance of VEGF}

VEGF's trophic activities are critical to maintaining cardiovascular, renal, and nervous tissues. Conditionally knocking out Vegfa in adult mouse retinal pigment epithelial (RPE) cells led to rapid ablation of the choriocapillaris followed by photoreceptor dysfunction and vision loss (2, 3). Astrocyte/neuron-derived VEGF is also important for developing and maintaining the retinal vasculature (4).

Systemic VEGF inhibition is likely to cause cardiovascular complications, such as hypertension and arterial thromboembolic events, or renal dysfunction (5). VEGF induces nitric oxide (NO) synthase expression by endothelial cells, resulting in the release of NO (a vasodilator) VEGF inhibition may lead to endothelial dysfunction, particularly in patients with increased hypertension or cardiovascular risk (6). Moreover, podocyte-derived VEGF is required for health and maintenance of the adjacent glomerular endothelium; its loss is associated with glomerular thrombotic microangiopathy $(7,8)$.

\section{Ocular effects of intravitreal anti-VEGF}

Geographic atrophy. AMD-associated geographic atrophy (GA) is characterized by progressive and irreversible loss of photoreceptors, RPE, and choriocapillaris. Five years of follow-up in the Comparison of AMD Treatments Trials showed that, among the 1011 participants who did not have GA at baseline and had follow-up images gradable for $\mathrm{GA}$, the cumulative incidence was $12 \%$ at one year, $17 \%$ at two years, and $38 \%$ at five years (9). Both the IVAN and HARBOR trials showed an increased risk of GA in eyes treated for two years with monthly dosing compared with

Conflict of interest: The authors have declared that no conflict of interest exists.

those receiving treatment as needed (10, 11). The SEVEN-UP study that followed ranibizumab treatment for neovascular AMD for up to eight years showed fundus autofluorescence-detected macular atrophy in $98 \%$ of eyes (12). Thus, emerging evidence suggests that anti-VEGF treatment can potentially increase the development of GA. Similarly, laboratory studies show that intravitreal bevacizumab injection into rats or nonhuman primates reduced fenestration of the normal choriocapillaris, which can lead to GA (13). VEGF deletion in mouse RPE was characterized by choriocapillaris atrophy and RPE/ Bruch's membrane abnormalities, similar to that observed in GA $(2,3)$.

Ocular hypertension. Injection of anti-VEGF into the eye is associated with short-term ocular hypertension caused by a transient, volume-dependent elevation of intraocular pressure (IOP) that typically resolves within 30 to 60 minutes. However, post hoc analysis of several clinical trials indicates that a small portion of patients (3\% to $11 \%)$ receiving repeated anti-VEGF injections developed ocular hypertension lasting several months or longer (14). Risk factors for chronic ocular hypertension were the total number of injections, a higher frequency of injection, and preexisting glaucoma. A significant decrease in outflow facility in patients receiving a higher number of intravitreal injections was also reported, perhaps explained by microparticle obstruction, toxic/inflammatory effects on trabecular meshwork, or loss of VEGF-mediated trabecular meshwork-secreted factors that regulate conventional outflow facility in response to mechanical stress (14). To date, there does not appear to be any long-term damage to the nerve fiber layer, although this should be monitored.

Less common adverse events. Anterior ischemic optic neuropathy, retinal vascular occlusions, hemorrhagic macular infarction, and development/exacerba- 
tion of ocular ischemic syndrome have been reported as rare adverse events (AEs) after intravitreal injection of anti-VEGF agents (15). While there are reports of decreased retrobulbar blood flow parameters, retinal arteriolar vasoconstriction, and worsening of macular ischemia after intravitreal injection of anti-VEGF agents, further studies are needed to understand the association between these effects and VEGF blockage (16). Other ocular AEs associated with anti-VEGF agents include traction retinal detachment (TRD) in PDR, RPE tears in choroidal neovascularization, and polypoidal choroidal vasculopathy. In a retrospective series, $5.2 \%$ of eyes developed or had progression of TRD following intravitreal bevacizumab injection for PDR (17). The incidence of RPE tears is inconsistently observed, with one study showing no significant increase with antiVEGF treatments while another observed that $7.9 \%$ of treatment-naive eyes treated with three monthly ranibizumab injections developed RPE tears by 12 months (18, 19). A higher risk of RPE tears was seen in patients with fibrovascular pigment epithelial detachment (19).

\section{Systemic effects of intravitreal anti-VEGF}

Systemic pharmacokinetics of intravitreal anti-VEGF. Although the dosage of antiVEGF for intravitreal injections is more than 100-fold lower than that used in oncology, aflibercept and bevacizumab injected intravitreally results in serum drug levels above $\mathrm{IC}_{50}$ concentrations (20). Ranibizumab (an Fab-based drug) is removed from the bloodstream more quickly than bevacizumab (intact immunoglobulin) or aflibercept because it has a single Fab fragment without the Fc region (20). Intravitreal aflibercept or bevacizumab treatment significantly reduced plasma-free VEGF relative to baseline, with aflibercept suppressing plasma-free VEGF levels below the lower detectable limit as early as three hours after injection and lasting more than seven days (20).

Systemic adverse effects. Two of the most common AEs of systemic bevacizumab therapy in oncology are proteinuria (18\% of patients) and hypertension (25.3\%; ref. 5). Human pathological studies and animal models using podocyte-specific VEGF deletion suggest that VEGF protects both the retinal and glomerular microvasculature, not only through VEGFR2-mediated vasculotrophism, but also through modulation of local proteins that could protect against complement-mediated damage (7, 8). The association of intravitreal administration of anti-VEGF with renal dysfunction, (e.g., worsening proteinuria in chronic kidney disease patients; ref. 21), is largely anecdotal to date and limited to case reports.

Hypertension is associated with the use of VEGF antagonists in oncology, although the mechanism for this remains unknown. Similarly, intravitreal bevacizumab injection in hypertensive patients significantly increases the risk for increasing blood pressure, while intravitreal ranibizumab does not (22). Although hypertension is a risk factor for cardio-/cerebrovascular accidents (CVA), the relationship between intravitreal anti-VEGF and the risk of these events is still controversial. In most clinical trials using intravitreal anti-VEGF therapy, the incidence of CVA, myocardial infarction (MI), and death is not significantly elevated, but these studies are underpowered for assessing small differences in rare events (23). Similarly, the incidence of systemic AEs did not differ between various anti-VEGF drugs, although one diabetic macular edema (DME) trial reported more arterial thrombotic events with ranibizumab versus aflibercept (24). Nevertheless, for some risk populations, such as patients with diabetes or a history of recent MI or CVA, there may be an increased risk with sustained suppression of systemic VEGF levels. One meta-analysis reported an increase in the risk of CVA and vascular death in diabetic patients receiving monthly anti-VEGF agents for two years (25). Moreover, the risk of MI after intravitreal bevacizumab injections was significantly associated with a history of CVA or MI (26).

Anti-VEGF therapy has emerged as a preferred treatment of infants with retinopathy of prematurity (ROP), a population at particularly high risk for sustained VEGF suppression due to VEGF's role in organogenesis and neurodevelopment. In ROP infants treated with bevacizumab, serum VEGF levels were significantly suppressed for up to 2 months after injection (27). Serum VEGF levels were less affected after intravitreal ranibizumab than after bevacizumab (27), suggesting that ranibi- zumab may be safer in infants. Studies of neurodevelopmental outcomes after intravitreal bevacizumab treatment for ROP have variable outcomes, with one study showing no difference in mean cognitive, language, or motor scores, while another reported lower median motor composite scores and 3.1 times higher incidence of neurodevelopmental disabilities in bevacizumab-treated infants. However, as these studies were not randomized, further research is needed to distinguish between prematurity itself and anti-VEGF treatment as the causative factor for neurodevelopmental abnormalities (28).

\section{Concluding remarks}

Systemic and ocular complications associated with intravitreal anti-VEGF therapies are uncommon in the general population, but careful observation and continuous assessment of dosing and frequency should be routine for at-risk populations. Postmarketing surveillance of the serious AEs are difficult because the medical team caring for a patient with a serious medical condition would not typically associate it with injections of VEGF antagonists. In AMD patients, VEGF antagonists do not prevent underlying disease progression and may accelerate GA development. Diabetics, elderly patients with widespread ischemic disease, and premature infants may be at particularly high risk for exacerbation of their underlying neurovascular dysfunction and, as such, should be carefully monitored if placed on anti-VEGF therapy. The next generation of more potent, longer-lasting anti-VEGF therapies will heighten concerns about potential AEs. Completely neutralizing vasculo/ neurotrophic activities of VEGF with these drugs may severely compromise collateralization that is a critical component of ischemic myocardial and CNS disease in the at-risk populations and lead to serious AEs in an elderly and/or critically ill group of patients. Combination therapies that reduce the dose of VEGF antagonists and minimize the risk of compensatory upregulation of other, non-VEGF-driven angiogenic pathways may provide lower risk for high-risk patients.

\section{Acknowledgments}

Work from the author's laboratory (MF) was supported by grants from the Lowy 
Medical Research Institute, the National Eye Institute, the California Institute for Regenerative Medicine, and the Scripps Clinical Medical Group. AUO is supported by grants from the Manpei Suzuki Diabetes Foundation and the Japan Society for the Promotion of Science Grants-in-Aid for Scientific Research (JSPS KAKENHI) (grant 17K16984). We are grateful for the constructive comments and suggestions by our colleagues in the Friedlander lab at The Scripps Research Institute, the Lowy Medical Research Institute, and the Scripps Clinical Medical Group as well as our clinical colleagues in the MacTel project who provided helpful advice in putting this viewpoint together.

Address correspondence to: Martin Friedlander, 10550 North Torrey Pines Road, La Jolla, California 92037, USA. Phone: 858. 784.2930; Email: friedlan@scripps.edu.

1. Gragoudas ES, Adamis AP, Cunningham ET, Feinsod M, Guyer DR, VEGF Inhibition Study in Ocular Neovascularization Clinical Trial Group. Pegaptanib for neovascular agerelated macular degeneration. $N$ Engl J Med. 2004;351(27):2805-2816.

2. Saint-Geniez M, Kurihara T, Sekiyama E, Maldonado AE, D’Amore PA. An essential role for RPE-derived soluble VEGF in the maintenance of the choriocapillaris. Proc Natl Acad Sci US A. 2009;106(44):18751-18756

3. Kurihara T, et al. Hypoxia-induced metabolic stress in retinal pigment epithelial cells is sufficient to induce photoreceptor degeneration. Elife. 2016;5:e14319.

4. Usui Y, et al. Neurovascular crosstalk between interneurons and capillaries is required for vision. J Clin Invest. 2015;125(6):2335-2346.

5. Zhao T, Wang X, Xu T, Xu X, Liu Z. Bevacizumab significantly increases the risks of hypertension and proteinuria in cancer patients: A systematic review and comprehensive meta-analysis. Oncotarget. 2017;8(31):51492-51506.
6. Olsson AK, Dimberg A, Kreuger J, ClaessonWelsh L. VEGF receptor signalling - in control of vascular function. Nat Rev Mol Cell Biol. 2006;7(5):359-371.

7. Eremina V, et al. VEGF inhibition and renal thrombotic microangiopathy. N Engl J Med. 2008;358(11):1129-1136.

8. Keir LS, et al. VEGF regulates local inhibitory complement proteins in the eye and kidney. J Clin Invest. 2017;127(1):199-214.

9. Grunwald JE, et al. Incidence and growth of geographic atrophy during 5 years of comparison of age-related macular degeneration treatments trials. Ophthalmology. 2017;124(1):97-104.

10. Chakravarthy U, et al. Alternative treatments to inhibit VEGF in age-related choroidal neovascularisation: 2-year findings of the IVAN randomised controlled trial. Lancet. 2013;382(9900):1258-1267.

11. Sadda SR, Tuomi LL, Ding B, Fung AE, Hopkins JJ. Macular atrophy in the HARBOR study for neovascular age-related macular degeneration. Ophthalmology. 2018;125(6):878-886.

12. Rofagha S, Bhisitkul RB, Boyer DS, Sadda SR, Zhang K, SEVEN-UP Study Group. Seven-year outcomes in ranibizumab-treated patients in ANCHOR, MARINA, and HORIZON: a multicenter cohort study (SEVEN-UP). Ophthalmology. 2013;120(11):2292-2299.

13. Peters $S$, et al. Ultrastructural findings in the primate eye after intravitreal injection of bevacizumab. Am JOphthalmol. 2007;143(6):995-1002.

14. Bracha P, Moore NA, Ciulla TA, WuDunn D, Cantor LB. The acute and chronic effects of intravitreal anti-vascular endothelial growth factor injections on intraocular pressure: A review. Surv Ophthalmol. 2018;63(3):281-295.

15. Falavarjani KG, Nguyen QD. Adverse events and complications associated with intravitreal injection of anti-VEGF agents: a review of literature. Eye (Lond). 2013;27(7):787-794.

16. Manousaridis K, Talks J. Macular ischaemia: a contraindication for anti-VEGF treatment in retinal vascular disease? Br JOphthalmol. 2012;96(2):179-184.

17. Arevalo JF, et al. Tractional retinal detachment following intravitreal bevacizumab (Avastin) in patients with severe proliferative diabetic retinopathy. Br JOphthalmol. 2008;92(2):213-216.

18. Cunningham ET, Feiner L, Chung C, Tuomi L,
Ehrlich JS. Incidence of retinal pigment epithelial tears after intravitreal ranibizumab injection for neovascular age-related macular degeneration. Ophthalmology. 2011;118(12):2447-2452.

19. Cho HJ, et al. Retinal pigment epithelial tear after intravitreal ranibizumab treatment for neovascular age-related macular degeneration. Retina (Philadelphia, Pa). 2016;36(10):1851-1859.

20. Avery RL, et al. Systemic pharmacokinetics and pharmacodynamics of intravitreal aflibercept, bevacizumab, and ranibizumab. Retina (Philadelphia, Pa). 2017;37(10):1847-1858.

21. Hanna RM, et al. Three patients with injection of intravitreal vascular endothelial growth factor inhibitors and subsequent exacerbation of chronic proteinuria and hypertension. Clin Kidney J. 2019;12(1):92-100.

22. Sengul A, et al. Short-term effects of intravitrea ranibizumab and bevacizumab administration on 24-h ambulatory blood pressure monitoring recordings in normotensive patients with age-related macular degeneration. Eye (Lond). 2017;31(5):677-683.

23. Thulliez M, et al. Cardiovascular events and bleeding risk associated with intravitreal antivascular endothelial growth factor monoclonal antibodies: systematic review and meta-analysis. JAMA Ophthalmol. 2014;132(11):1317-1326.

24. Low A, et al. Comparative effectiveness and harms of intravitreal antivascular endothelial growth factor agents for three retinal conditions: a systematic review and meta-analysis. $\mathrm{Br} \mathrm{JOPh-}$ thalmol. 2019;103(4):442-451.

25. Avery RL, Gordon GM. Systemic safety of prolonged monthly anti-vascular endothelial growth factor therapy for diabetic macular edema: a systematic review and meta-analysis. JAMA Ophthalmol. 2016;134(1):21-29.

26. Kwon JW, Jee D, La TY. The association between myocardial infarction and intravitreal bevacizumab injection. Medicine (Baltimore). 2018;97(13):e0198.

27. Wu WC, et al. Serum vascular endothelial growth factor after bevacizumab or ranibizumab treatment for retinopathy of prematurity. Retina. 2017;37(4):694-701.

28. Darwish D, et al. Anti-vascular endothelial growth factor and the evolving management paradigm for retinopathy of prematurity. Asia Pac JOphthalmol (Phila). 2018;7(3):136-144. 